\title{
Estimation of $\mathrm{P}(Y<X)$ for a Two-parameter Bathtub Shaped Failure Rate Distribution
}

\author{
Ammar M. Sarhan ${ }^{1}$, Bruce Smith ${ }^{1} \&$ David C. Hamilton ${ }^{1}$ \\ ${ }^{1}$ Department of Mathematics and Statistics, Dalhousie University, Halifax NS B3H 4R2, Canada \\ Correspondence: Ammar M. Sarhan, Department of Mathematics and Statistics, Dalhousie University, Halifax NS \\ B3H 4R2, Canada. Tel: 1-902-266-6423. E-mail: asarhan@mathstat.dal.ca
}

Received: February 5, 2015 Accepted: February 26, 2015 Online Published: March 25, 2015

doi:10.5539/ijsp.v4n2p33 URL: http://dx.doi.org/10.5539/ijsp.v4n2p33

\begin{abstract}
This paper deals with the estimation of reliability $R=P[Y<X]$ when $X$ and $Y$ are two independent random variables with a two-parameter bathtub shaped failure rate distribution with the same second shape parameter. Likelihood and Bayesian methods are proposed to make inferences about $R$. We obtain the likelihood interval and asymptotic confidence interval for $R$, and we consider Bayesian point estimates of $R$ under both absolute and squared error loss, using either gamma or uniform priors for the three unknown model parameters. An equal tail Bayesian credible interval for $R$ is investigated. Analysis of a real data set is presented for illustrative purposes, and Monte Carlo simulations are performed to compare: (1) the performance of Bayes estimates under two different loss functions; and (2) the maximum likelihood and Bayesian methods.
\end{abstract}

Keywords: maximum likelihood estimator, Fisher information matrix, Bayes estimator, reliability.

\section{Introduction}

Chen (2000) reinvestigates a two-parameter bathtub shaped failure rate distribution which was originally considered by Gurvich et al. (1997). The cumulative distribution function (cdf) of this distribution with parameters $\lambda>0$ and $\beta>0$ is, Chen (2000),

$$
F(x ; \lambda, \alpha)=1-e^{\lambda\left(1-e^{x^{\alpha}}\right)}, \quad x>0 .
$$

The corresponding hazard rate function is

$$
h(x ; \lambda, \alpha)=\alpha \lambda x^{\alpha-1} e^{x^{\alpha}}, \quad x>0,
$$

and the survival function is

$$
S(x ; \alpha, \beta)=e^{\lambda\left(1-e^{x^{\alpha}}\right)}, \quad x>0 .
$$

The probability density function (pdf) is

$$
f(x ; \alpha, \beta)=\alpha \lambda x^{\alpha-1} e^{x^{\alpha}} e^{\lambda\left(1-e^{x^{\alpha}}\right)}, x>0 .
$$

Here $\alpha$ and $\lambda$ are both shape parameters. Henceforth, we will denote this distribution by TPBT $(\lambda, \alpha)$ or TPBT.

Due to the convenient structure of the TPBT distribution, it can be used in analyzing many lifetime data sets. The failure rate function takes a bathtub shape when $\alpha<1$ and is increasing if $\alpha \geq 1$, Chen (2000). The TPBT distribution is the only known two parameter distribution with a bathtub shaped failure rate function that has exact joint confidence regions for the parameters Chen (2000). Sarhan et al. (2012) discussed Bayes estimation of the two parameters of the TPBT distribution.

In a stress-strength model, the stress $Y$ and the strength $X$ are treated as random variables and the reliability, $R$, of a component during a given period is taken to be the probability that its strength exceeds the stress during the entire interval. Due to its practical importance, the estimation of $R=P(Y<X)$ has attracted the attention of many authors. The maximum likelihood estimate (MLE) of $R$, under the assumption that $X$ and $Y$ are independent and normally distributed, is derived by Church and Harris (1970). The uniformly minimum variance unbiased estimate of $R$ under the same assumption is obtained by Downtown (1973). Recently, Kundu and Gupta (2005) have considered estimation of $P(Y<X)$ when $X$ and $Y$ are independent generalized exponential random variables. 
Kundu and Gupta (2006) studied the problem when $X$ and $Y$ are independent random variables having Weibull distributions with different scale parameters but having the same shape parameter, and Kakade et al. (2008) considered the case when $X$ and $Y$ are independent exponentiated Gumbel distributed random variables. Rezae et al. (2010) studied the estimation problem for $P(Y<X)$ when $X$ and $Y$ are independent and follow generalized Pareto distributions with common scale parameter. Ali (2013) discussed Bayes estimation of $P(Y<X)$, using different loss functions, when $X$ and $Y$ are independent and Lindley distributed. Sharma et al. (2014) studied the same problem when $X$ and $Y$ are independent and follow the inverse Lindley distribution. Kumar et al. (2014) studied maximum likelihood maximum likelihood and Bayes estimates for the parameter $P(Y<X)$ when $X$ and $Y$ are independent and Lindley distributed.

Our main aim in this paper is to focus on inferences for $R=P[Y<X]$ when $X$ and $Y$ are two independent but not identically distributed random variables with the TPBT distribution. It is assumed that $X$ follows $\operatorname{TPBT}(\lambda, \alpha)$ and $Y$ follows $\operatorname{TPBT}(\gamma, \alpha)$. Estimation of $R$ is very common in the statistical and reliability literature. For example, if $X$ is the strength of a component which is subject to a stress $Y$, then $R$ is a measure of system performance and arises in the context of mechanical reliability of a system. The system fails if and only if at any time the applied stress is greater than its strength. We derive the MLE of $R$ and obtain its asymptotic distribution, which is used to construct an asymptotic confidence interval for $R$. We approximate Bayes estimators and credible intervals for $R$ using Markov Chain Monte Carlo (MCMC) sampling. The Bayes and non-Bayes methods are compared using Monte Carlo simulations and a data set is analysed for illustrative purposes.

The Bayesian approach requires integration over the posterior distribution and this is often impossible to carry out analytically. In such cases, MCMC techniques can be applied to draw samples from the posterior distribution. The MCMC methodology provides a convenient and efficient method to sample complex, highly-dimensional distributions.

The rest of the paper is organized as follows. Section 2 presents the MLE of the unknown parameters and the reliability. The asymptotic confidence intervals of the unknown parameters and reliability are discussed in Section 3. Sections 4 discusses Bayesian inference. A complete analysis of a real data set is given in Section 5 for illustrative purposes. Section 6 gives a simulation study, and Section 7 concludes the paper.

\section{Maximum Likelihood Estimate of $\mathbf{R}$}

Assume that there are two independent random variables $Y$ and $X$ such that $X \sim \operatorname{TPBT}(\lambda, \alpha)$ and $Y \sim \operatorname{TPBT}(\gamma, \alpha)$. Therefore,

$$
\begin{aligned}
R=P(Y<X) & =\int_{0}^{\infty} \int_{0}^{x} \lambda \gamma \alpha^{2}(x y)^{\alpha-1} e^{x^{\alpha}+y^{\alpha}} e^{\lambda\left(1-e^{\alpha^{\alpha}}\right)+\gamma\left(1-e^{\gamma^{\alpha}}\right)} d y d x \\
& =1-\int_{0}^{\infty} \lambda \alpha x^{\alpha-1} e^{x^{\alpha}} e^{(\lambda+\gamma)\left(1-e^{\alpha^{\alpha}}\right)} d x \\
& =\frac{\gamma}{\lambda+\gamma} .
\end{aligned}
$$

To obtain the MLE of $R$, we first derive the MLE of the two parameters $\gamma$ and $\lambda$ and then use the invariance property. Now, suppose $X_{1}, X_{2}, \cdots, X_{n}$ is an independent random sample from $\operatorname{TPBT}(\lambda, \alpha)$ and $Y_{1}, Y_{2}, \cdots, Y_{m}$ is an independent random sample from $\operatorname{TPBT}(\gamma, \alpha)$. Let $\theta=(\alpha, \lambda, \gamma)^{\prime}$ denote a vector of the three unknown parameters. The likelihood function, $L=L(\theta)$, of $\theta=(\alpha, \lambda, \gamma)^{\prime}$ is

$$
L(\alpha, \lambda, \gamma) \propto \alpha^{n+m} \lambda^{n} \gamma^{m}\left(\prod_{i=1}^{n} x_{i} \prod_{i=1}^{m} y_{i}\right)^{\alpha-1} e^{\sum_{i=1}^{n} x_{i}^{\alpha}+\sum_{i=1}^{m} y_{i}^{\alpha}} e^{\lambda \sum_{i=1}^{n}\left(1-e^{x_{i}^{\alpha}}\right)+\gamma \sum_{i=1}^{m}\left(1-e_{i}^{\gamma^{\alpha}}\right)} .
$$

Thus, the log-likelihood function, $\mathcal{L}=\mathcal{L}(\theta)=\mathcal{L}(\alpha, \lambda, \gamma)$, is

$$
\begin{gathered}
\mathcal{L}(\alpha, \lambda, \gamma) \propto \quad(n+m) \ln \alpha+n \ln \lambda+m \ln \gamma+(\alpha-1)\left(\sum_{i=1}^{n} \ln x_{i}+\sum_{i=1}^{m} \ln y_{i}\right) \\
+\sum_{i=1}^{n} x_{i}^{\alpha}+\sum_{i=1}^{m} y_{i}^{\alpha}+\lambda \sum_{i=1}^{n}\left(1-e^{x_{i}^{\alpha}}\right)+\gamma \sum_{i=1}^{m}\left(1-e^{y_{i}^{\alpha}}\right) .
\end{gathered}
$$


The likelihood equations are

$$
\begin{aligned}
& \frac{\partial \mathcal{L}}{\partial \alpha}=\frac{n+m}{\alpha}+\sum_{i=1}^{n}\left(1+x_{i}^{\alpha}\right) \ln x_{i}-\lambda \sum_{i=1}^{n} e^{x_{i}^{\alpha}} x_{i}^{\alpha} \ln x_{i}+\sum_{i=1}^{m}\left(1+y_{i}^{\alpha}\right) \ln y_{i}-\gamma \sum_{i=1}^{m} e^{y_{i}^{\alpha}} y_{i}^{\alpha} \ln y_{i}=0, \\
& \frac{\partial \mathcal{L}}{\partial \lambda}=\frac{n}{\lambda}+\sum_{i=1}^{n}\left(1-e^{x_{i}^{\alpha}}\right)=0, \\
& \frac{\partial \mathcal{L}}{\partial \gamma}=\frac{m}{\gamma}+\sum_{i=1}^{m}\left(1-e^{y_{i}^{\alpha}}\right)=0 .
\end{aligned}
$$

From (5) and (6), we obtain the MLE of $\lambda$ and $\gamma$ as functions of $\alpha$, say $\hat{\lambda}(\alpha)$ and $\hat{\gamma}(\alpha)$ respectively, as

$$
\hat{\lambda}(\alpha)=\frac{n}{-n+\sum_{i=1}^{n} e^{x_{i}^{\alpha}}},
$$

and

$$
\hat{\gamma}(\alpha)=\frac{m}{-m+\sum_{i=1}^{m} e^{y_{i}^{\alpha}}} .
$$

Substituting $\hat{\lambda}(\alpha)$ and $\hat{\gamma}(\alpha)$ into (3), we get the profile log-likelihood function for $\alpha$ as

$$
\begin{aligned}
\mathcal{L}(\alpha, \hat{\lambda}(\alpha), \hat{\gamma}(\alpha))= & -(n+m)+n \ln n+m \ln m+(n+m) \ln \alpha+(\alpha-1)\left[\sum_{i=1}^{n} \ln x_{i}+\sum_{i=1}^{m} \ln y_{i}\right] \\
& +\sum_{i=1}^{n} x_{i}^{\alpha}+\sum_{i=1}^{m} y_{i}^{\alpha}-n \ln \sum_{i=1}^{n}\left(e^{x_{i}^{\alpha}}-1\right)-m \ln \sum_{i=1}^{m}\left(e^{y_{i}^{\alpha}}-1\right) .
\end{aligned}
$$

Therefore, the MLE of $\alpha$, say $\hat{\alpha}$, can be obtained by maximizing (9) with respect to $\alpha$. It can be shown that the $\hat{\alpha}$ can be obtained as a solution of a non-linear equation of the form

$$
h(\alpha)=\alpha,
$$

where

$$
h(\alpha)=\frac{n+m}{\frac{n \sum_{i=1}^{n} e^{\alpha_{i}^{\alpha}} x_{i}^{\alpha} \ln x_{i}}{-n+\sum_{i=1}^{n} e_{i}^{\alpha_{i}}}+\frac{m \sum_{i=1}^{m} e_{i}^{\alpha_{i}^{\alpha}} y_{i}^{\alpha} \ln y_{i}}{-m+\sum_{i=1}^{m} e_{i}^{\alpha_{i}^{\alpha}}}-\sum_{i=1}^{n}\left(1+x_{i}^{\alpha}\right) \ln x_{i}-\sum_{i=1}^{m}\left(1+y_{i}^{\alpha}\right) \ln y_{i}} .
$$

A very simple and effective iterative procedure $\alpha^{(j+1)}=h\left(\alpha^{(j)}\right)$ can be used, where $\alpha^{(j)}$ is the $j$ th iterate of $\hat{\alpha}$. The iteration procedure should be stopped when $\left|\alpha^{(j+1)}-\alpha^{(j)}\right|$ is sufficiently small. Once we obtain $\hat{\alpha}, \hat{\lambda}$ and $\hat{\gamma}$ can be calculated from (7) and (8) respectively, and the MLE of $R$ is

$$
\hat{R}=\frac{\hat{\gamma}}{\hat{\gamma}+\hat{\lambda}} \text {. }
$$

\section{Confidence intervals for $\mathbf{R}$}

Note that, $\hat{\alpha}, \hat{\lambda}$ and $\hat{\gamma}$ are not in explicit form. Further, it is not possible to obtain the variances $\hat{\theta}=(\hat{\alpha}, \hat{\lambda}, \hat{\gamma})^{\prime}$ in an explicit form. We propose to use two approximate confidence intervals for $R$.

\subsection{Likelihood Interval}

The maximum log-relative likelihood function for $R, r_{\max }(R)$, is

$$
r_{\max }(R)=\mathcal{L}(\hat{\alpha}(R), \hat{\lambda}=(1 / R-1) \hat{\gamma}(R), \hat{\gamma}(R))-\mathcal{L}(\hat{\alpha}, \hat{\lambda}, \hat{\gamma}),
$$

where

$$
\hat{\gamma}(R)=\frac{n+m}{(1 / R-1)\left(\sum_{i=1}^{n} e^{\hat{\alpha}_{i}^{(R)}}-n\right)+\sum_{j=1}^{m} e^{\hat{y}_{j}^{\hat{\alpha}(R)}}-m}
$$

and $\hat{\alpha}(R)$ is the solution of the following equation in $\alpha$ for a given $R$

$$
0=\frac{1}{\alpha}+\frac{1}{n+m} \sum_{i=1}^{n}\left(1+x_{i}^{\alpha}\right) \ln x_{i}+\frac{1}{n+m} \sum_{i=1}^{n}\left(1+y_{j}^{\alpha}\right) \ln y_{j}-\frac{(1 / R-1) \sum_{i=1}^{n} x_{i}^{\alpha} e^{x_{i}^{\alpha}} \ln x_{i}+\sum_{j=1}^{m} y_{j}^{\alpha} e^{y_{j}^{\alpha}} \ln y_{j}}{(1 / R-1)\left(\sum_{i=1}^{n} e^{x_{i}^{\alpha}}-n\right)+\sum_{j=1}^{m} e^{y_{j}^{\alpha}}-m}
$$


A $100 p \%$ likelihood interval (LI) for $R$ is the set of all possible real solutions, in $R$, of the following inequality

$$
r_{\max }(R) \geq \ln (p), 0<p<1 .
$$

This inequality has no closed solution in $R$, so the interval must be obtained numerically. The $100 p \%$ LI approximates a $(1-\vartheta) 100 \%$ CI when $p=\exp \left\{-\frac{1}{2} \chi_{1}^{2}(\vartheta)\right\}$, where $\chi_{1}^{2}(\vartheta)$ is the upper $\vartheta$ quantile of $\chi_{1}^{2}$.

\subsection{Asymptotic Confidence Interval}

The MLE of the vector of unknown parameters $\theta=(\alpha, \lambda, \gamma)^{\prime}$ is asymptotically normally distributed with mean of true $\theta$ and variance-covariance matrix $\mathfrak{I}^{-1}$, and is the inverse of the expected information matrix $\mathfrak{I}(\theta)$. $\mathfrak{I}(\theta)$ is consistently estimated by $\hat{\mathfrak{I}}=\mathfrak{I}(\hat{\theta})$, which is given in the Appendix. Let $\hat{\mathfrak{I}}^{j j}$ be the $(j, j)$-entry of $\hat{\mathfrak{I}}, j=1,2,3$, then the asymptotic $(1-\vartheta) 100 \%$ confidence intervals for $\theta_{j}, j=1,2,3$, are

$$
\hat{\theta}_{j} \pm Z_{\vartheta / 2} \sqrt{\hat{\mathfrak{I}}^{j j}}
$$

where $Z_{\vartheta / 2}$ is the upper $\vartheta / 2$ quantile of the standard normal distribution.

One can show that, $\hat{R}$ is asymptotically normally distributed with mean of $R$ and variance $\sigma_{R}^{2}$. $\sigma_{R}^{2}$ is consistently estimated by

$$
\hat{\mathfrak{I}}_{R}=\frac{1}{(\hat{\lambda}+\hat{\gamma})^{4}}\left[\hat{\gamma}^{2} \hat{\mathfrak{I}}^{22}-2 \hat{\lambda} \hat{\gamma} \hat{\mathfrak{I}}^{23}+\hat{\lambda}^{2} \hat{\mathfrak{I}}^{33}\right] .
$$

Therefore, an asymptotic $(1-\vartheta) 100 \%$ confidence interval for $R$ is

$$
\hat{R} \pm \frac{Z_{\vartheta / 2}}{(\hat{\lambda}+\hat{\gamma})^{2}} \sqrt{\hat{\gamma}^{2} \hat{\mathfrak{I}}^{22}-2 \hat{\lambda} \hat{\gamma} \hat{\mathfrak{I}}^{23}+\hat{\lambda}^{2} \hat{\mathfrak{I}}^{33}}
$$

\section{Bayesian Inference}

In this section, we discuss Bayesian methods for making inferences about $R=\frac{\gamma}{\lambda+\gamma}$.

We assume that the prior pdfs of the elements of $\theta=(\alpha, \lambda, \gamma)^{\prime}$ are independent, and that they are either gamma distributed $\pi(u) \propto \alpha^{a-1} e^{-b u}, u>0$ for fixed values of $a, b>0$, or that they are they are uniformly distributed on $(0, B)$, for some large $B$.

Using the likelihood function (2) and independent uniform priors, the joint posterior pdf of $\theta=(\alpha, \lambda, \gamma)^{\prime}$ is proportional to the likelihood function, while using independent gamma priors the joint posterior pdf $\theta$ is

$$
\begin{aligned}
\pi(\theta \mid \text { data }) \propto & \alpha^{a_{1}+n+m-1} \lambda^{a_{3}+n-1} \gamma^{a_{2}+m-1} \exp \left\{\alpha\left[-b_{1}+\sum_{i=1}^{n} \ln x_{i}+\sum_{i=1}^{m} \ln y_{i}\right]+\sum_{i=1}^{n} x_{i}^{\alpha}+\sum_{i=1}^{m} y_{i}^{\alpha}\right. \\
& \left.+\lambda\left[-b_{3}+\sum_{i=1}^{n}\left(1-e^{x_{i}^{\alpha}}\right)\right]+\gamma\left[-b_{2}+\sum_{i=1}^{m}\left(1-e^{y_{i}^{\alpha}}\right)\right]\right\}, \theta>0 .
\end{aligned}
$$

In either case the joint posterior density has a complicated form and it is unlikely that closed form inferences for the parameters $\alpha, \beta, \gamma$, or for the reliability $R$, are possible. As indicated by Gilks et al (1996), any feature of the posterior distribution is a legitimate candidate for Bayesian inference, for example, moments, quantiles, or highest posterior density regions. Such quantities can often be expressed in terms of posterior expectations of functions of $\theta$, and while the integrations in such expectations are typically difficult to be evaluate analytically, Markov Chain Monte Carlo (MCMC) sampling methods can often be used to approximate the integrals. In any event, Bayesian inferences are always based on the posterior distribution, and where analytical forms are not not available, inference is most often accomplished using samples from the posterior distribution. Good general references are Aykroyd (1998) and Gelman et al (2009).

We sampled from the posterior distribution $p(\theta \mid$ data) using the Metropolis-Hastings algorithm (Hastings, 1970), summarized as follows.

1. Choose an intitial value $\theta^{(0)}=\left(\alpha^{(0)}, \lambda^{(0)}, \gamma^{(0)}\right)^{\prime}$. 
2. For $t=1, \cdots, T$ repeat the following steps

$i$. Set $\theta=\theta^{(t-1)}$.

ii. Generate a candidate value $\theta^{*}$ from a proposal distribution $q\left(\theta^{*} \mid \theta\right)$.

iii. Set $\theta^{(t)}=\theta^{*}$ with probability $\min \left\{1, \frac{\pi\left(\theta^{*} \mid \text { data }\right) q\left(\theta \theta^{*}\right)}{\pi(\theta \mid \text { data }) q\left(\theta^{*} \mid \theta\right)}\right\}$, and otherwise set $\theta^{(t)}=\theta$.

Here $q$ is the transition probability matrix of a Markov chain whose support is the same as that of the likelihood function, and $q\left(\theta^{*} \mid \theta\right)$ is the probability of a transition from $\theta$ to $\theta^{*}$. Tierney (1994) provides an extensive discussion on the use of the Metropolis-Hastings and related algorithms for sampling posterior distributions, including the choice of the proposal distribution. Under quite general conditions the Metropolis-Hastings algorithm generates a sample path $\left\{\theta^{(t)}, t=0,1, \ldots T\right\}$ from an ergodic Markov Chain whose marginal distributions converge to the equilibrium distribution of the chain - in this case the posterior distribution from which we wish to sample - as $T \rightarrow \infty$. While there are many possible choices for the proposal distribution, in practice the choice of the proposal is important since a poor choice can delay the convergence towards the equilibrium distribution.

We generated proposals using an independence chain with proposals chosen as i.i.d. samples from $N_{3}\left(\hat{\theta}, \hat{\mathfrak{I}}^{-1}\right)$. The Bernstein-von Mises's theorem (Lecam, 1986) states that this is the limit distribution of the posterior $p(\theta \mid$ data), as $n \rightarrow \infty$. The idea of using an independence chain in which the proposed point is independent of the past and current states was suggested by Hastings (1970), among other proposal strategies.

In order to assess the sensitivity of inference to the choice of prior, we used two prior distributions with very different characteristics, either $\alpha, \lambda, \gamma, \sim$ i.i.d. $\mathrm{U}(0,100)$, or $\alpha, \lambda, \gamma, \sim$ i.i.d. gamma(.001, .001$)$.

The sampled values $\gamma^{(t)}=\theta_{3}^{(t)}$ and $\lambda^{(t)}=\theta_{2}^{(t)}$ are transformed to provide samples from $R$ as $\hat{R}^{(t)}=\frac{\gamma^{(t)}}{\lambda^{(t)}+\gamma^{(t)}}$, and these are used to carry out Bayesian inference for $R$.

\section{Application}

In this section we present a data analysis of the strength data reported by Badar and Priest [4]. The data represent the strength, measured in GPa, for single carbon fibers and impregnated 1000-carbon fiber tows. Single fibers were tested under tension at gauge lengths of $1 ; 10 ; 20$; and $50 \mathrm{~mm}$. Impregnated tows of 1000 fibers were tested at gauge lengths of 20;50;150 and $300 \mathrm{~mm}$. For illustrative purposes, we consider the single fibers of $20 \mathrm{~mm}$ (Data Set I) and $10 \mathrm{~mm}$ (Data Set II) in gauge length, with sample sizes $n=69$ and $m=63$, respectively. The data are presented below for convenience.

Data Set $1(X:)$

1.3121 .3141 .4791 .5521 .7001 .8031 .8611 .8651 .9441 .9581 .9661 .9972 .0062 .0212 .0272 .0552 .0632 .098

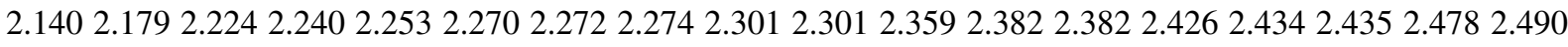

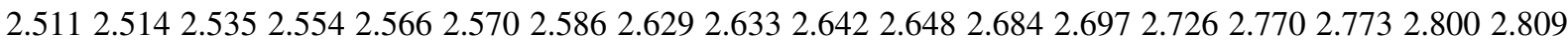

2.8182 .8212 .8482 .8802 .9543 .0123 .0673 .0843 .0903 .0963 .1283 .2333 .4333 .5853 .585

DATA SET $2(Y:)$

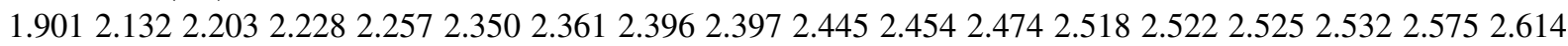
2.6162 .6182 .6242 .6592 .6752 .7382 .7402 .8562 .9172 .9282 .9372 .9372 .9772 .9963 .0303 .1253 .1393 .145 3.2203 .2233 .2353 .2433 .2643 .2723 .2943 .3323 .3463 .3773 .4083 .4353 .4933 .5013 .5373 .5543 .5623 .628 3.8523 .8713 .8863 .9714 .0244 .0274 .2254 .3955 .020

We fit the TPBT model to the two data sets separately, and present the estimated parameters of both generalized exponential (GE) and TPBT distributions, log-likelihood values $\mathcal{L}$, Kolmogorov-Smirnov (K-S) distances and corresponding $p$-values in Table 1. Also, the Akaike information criterion ( $\mathrm{AIC}=-2 \mathcal{L}+2 k, k$ is the number of model parameters) is computed for every model using the two data sets. Based on either the P-value or the AIC, the TPBT model fits quite well (and better than the GE model) to both the data sets. Empirical and fitted survivor functions are shown in Figure 1.

Table 1: MLE of the parameters, K-S, P-value, $\mathcal{L}$, and AIC for the two data sets.

\begin{tabular}{llccccc}
\hline Variable & Model & mles & K-S & P-value & $\mathcal{L}$ & AIC \\
\hline$X$ & TPBT & $(0.1516,1.3675)$ & 0.08471 & 0.6733 & -51.704 & 107.408 \\
& GE & $(1.8966,8.8284)$ & 0.10568 & 0.3965 & -56.669 & 117.383 \\
\hline$Y$ & TPBT & $(0.2809,1.0375)$ & 0.10126 & 0.5059 & -60.077 & 124.154 \\
& GE & $(1.6777,6.4538)$ & 0.17053 & 0.0452 & -64.195 & 132.390 \\
\hline
\end{tabular}


Assuming a common parameter $\alpha$ for the TPBT model, we obtained the MLEs $\hat{\alpha}=1.2015, \hat{\lambda}=0.042308$ and $\hat{\gamma}=0.012396$ leading to the MLE of $R$ as $\hat{R}=0.22661$. The inverse of the observed information matrix of $(\hat{\alpha}, \hat{\lambda}, \hat{\gamma})$ is

$$
\hat{\mathfrak{I}}^{-1}=\left(\begin{array}{rrr}
1.0113 \times 10^{-3} & -1.6217 \times 10^{-4} & -8.8439 \times 10^{-5} \\
-1.6217 \times 10^{-4} & 5.1948 \times 10^{-5} & 1.4182 \times 10^{-5} \\
-8.8439 \times 10^{-5} & 1.4182 \times 10^{-5} & 1.0173 \times 10^{-5}
\end{array}\right)
$$

Using (12) and (15), we obtained $\hat{\mathfrak{I}}_{R}=1.2638 \times 10^{-3}$. Therefore, using (11) and (13), we obtained the $14.7 \%$ LI (which approximates a 95\% CI) and the asymptotic $95 \%$ CI for $R$ as $(0.17984,0.28367)$ and $(0.15693,0.29628)$ respectively. The intervals are close, but not identical, reflecting the asymmetry of the maximum log-relative likelihood function $r_{\max }(R)$, which is plotted in Figure 2 together with the endpoints of the $14.7 \%$ LI for $R$.

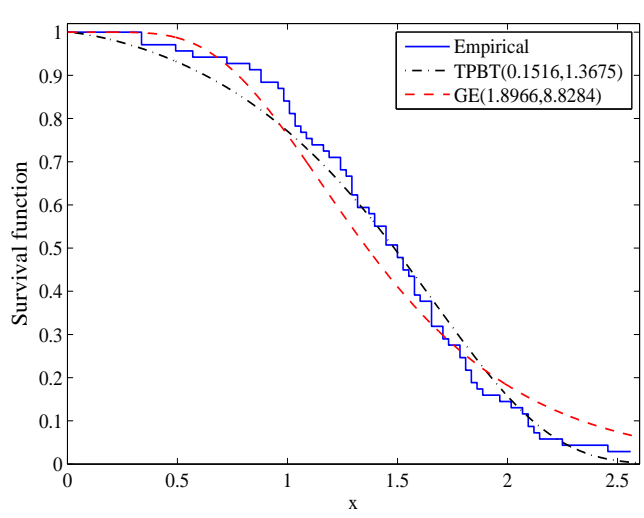

(a) Data 1

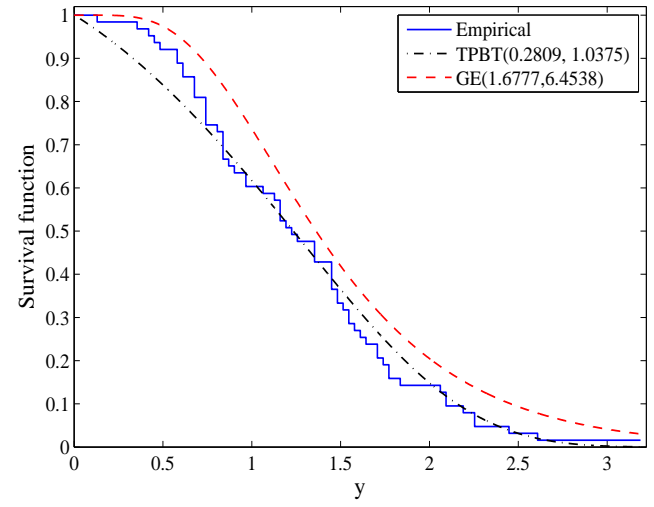

(b) Data 2

Figure 1. Empirical and fitted survival functions for the two data sets using GE and TPBT models.

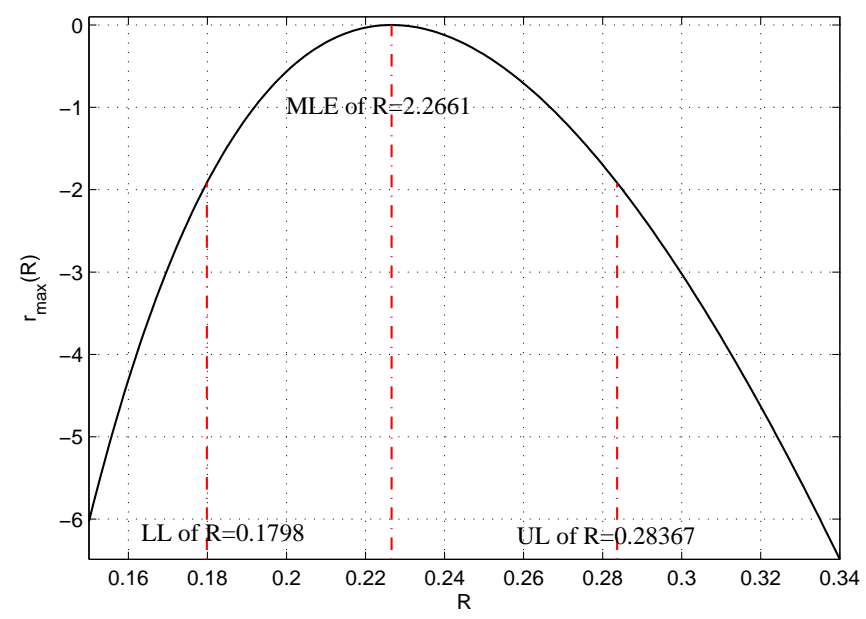

Figure 2. Max Log-relative likelihood function of $R$ along with the endpoints of $14.7 \%$ LI for $R$.

Figure 3a shows values of $\alpha, \lambda$ and $\gamma$ sampled from the joint posterior distribution assuming independent gamma(.001,.001) priors, after deleting an initial transient of 10,000 points and subsampling each 200 'th point. The empirical bivariate marginal distributions are also included in Figures $3 \mathrm{~b}, \mathrm{c}$ and $\mathrm{d}$ along with $p=0.01,01.47$ and 0.50 profile likelihood contours. There is a good agreement between the shape of the contours and the sample from the posterior indicating little dependence on the prior.

Figure 4 shows the 1000 sampled points from the marginal posterior distributions of $\alpha, \lambda, \gamma$ and $R$ calculated using gamma priors, and histograms of the empirical marginal posterior pdfs. The dashed lines on the left hand panels are positioned at the upper and lower limits of the $14.7 \%$ likelihood intervals. 
a)

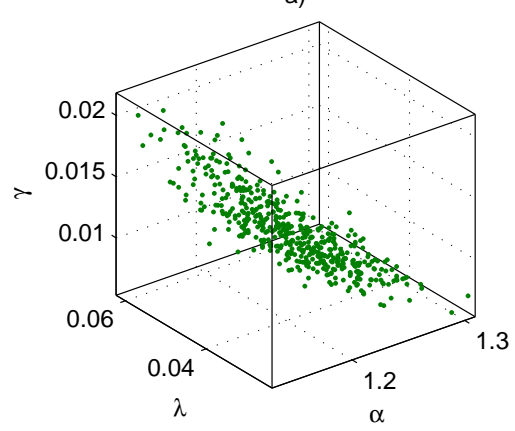

c)

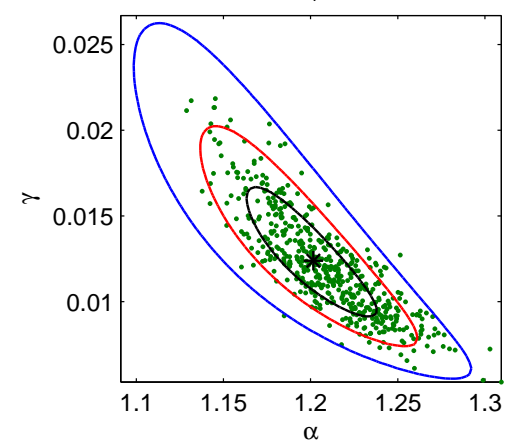

b)

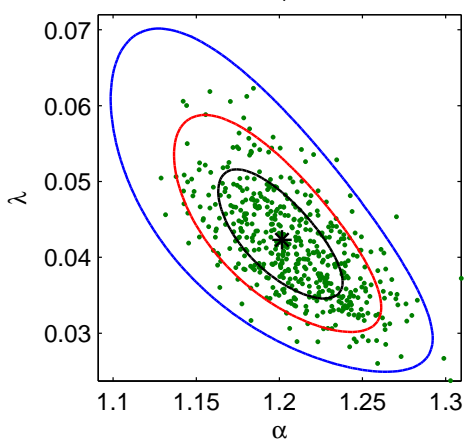

d)

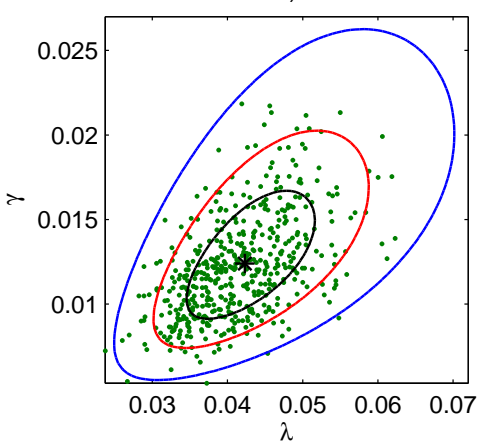

Figure 3. Scatter plots showing sample values (a) and marginal samples values and likelihood contours (b), (c) and (d). Contour levels are $p=0.01, p=0.147$ and $p=0.50 .{ }^{*}$ indicates the MLE.
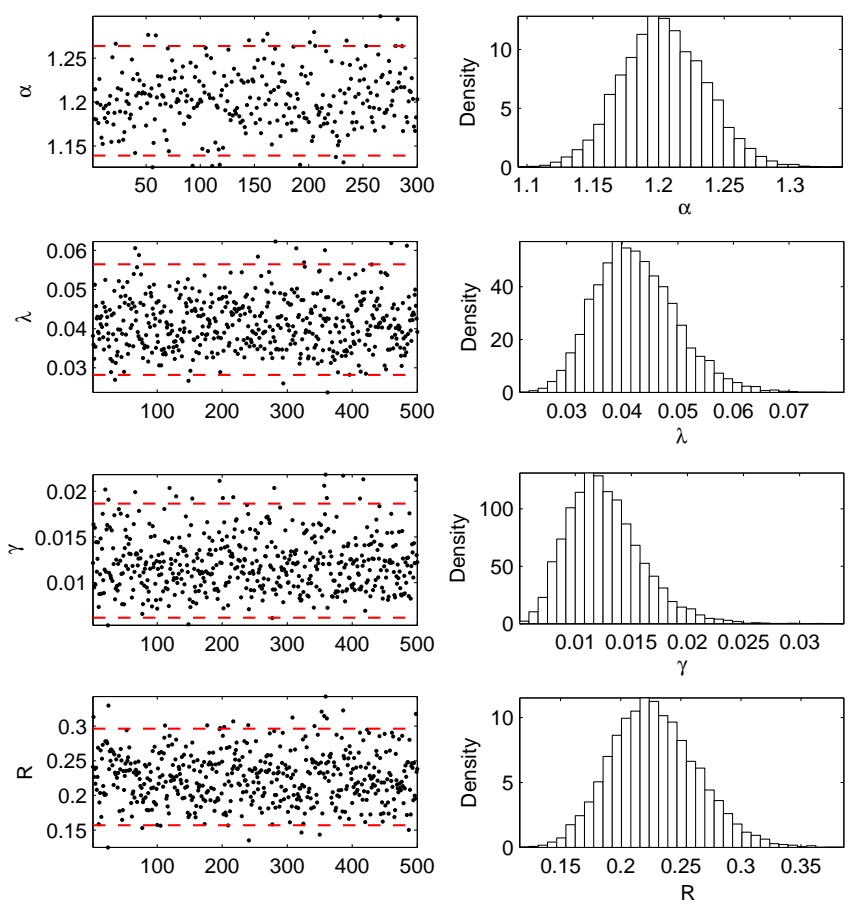

Figure 4. Simulated points from the marginal posterior distributions along with $14.7 \%$ LI's and empirical marginal posterior pdfs using gamma priors. 
In order to assess the sensitivity of Bayesian inferences for $\mathrm{R}$ to the choice of prior we ran the Metropolis-Hastings algorithm twice: with independent $\mathrm{U}(0,100)$ priors, and with independent gamma $(0.001,0.001)$ priors. The uniform distribution was meant to play the role of a non-informative prior, while the gamma prior is relatively informative, being heavily skewed to the right with a single high mode near 0 . We ran the sampler for 100,000 iterations and discarded the first 50,000 points as a transient. The remaining 50000 points were sub-sampled, retaining every 50th point, and the resulting sequences of 1000 sampled values of $R$ are shown in Figure 5, together with plots of the associated simulated empirical posterior pdf. These figures suggest that the choice of prior has little influence on inferences for $R$, and this is further evidenced in Table 2, which shows the estimated posterior means and medians.

Equal tail probability 0.95 credible intervals for $\mathrm{R}$ were calculated as the interval from the 2.5 'th to the $97.5^{\prime}$ th percentile of the empirical distribution of $\mathrm{R}$. The intervals, $(0.177,0.276)$ using the gamma prior, and $(0.180$, 0.277) using the uniform prior, again show little sensitivity to the choice of prior.
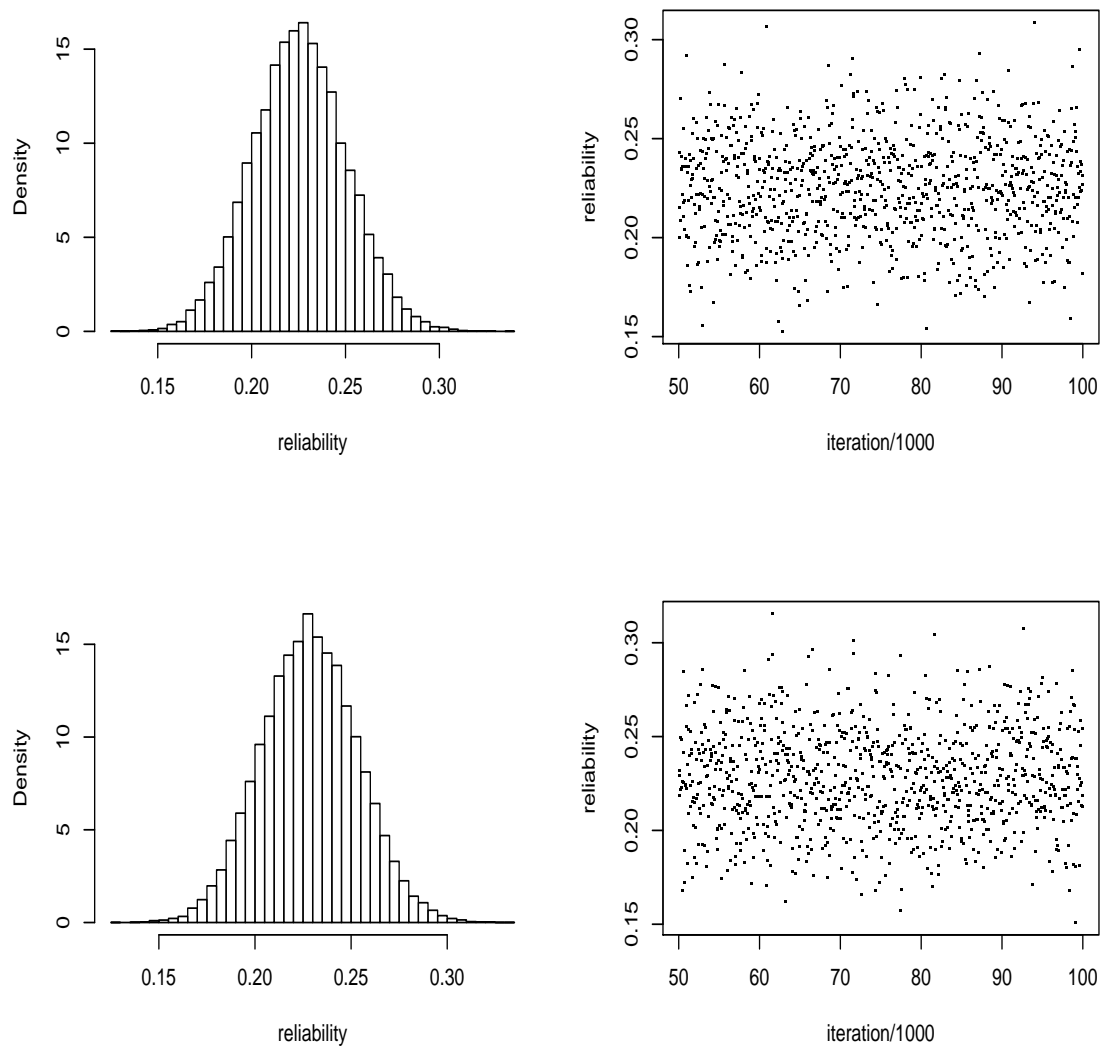

Figure 5. Simulated points and empirical posterior pdfs of $R$ using gamma (top row) and uniform (bottom row) priors.

Table 2. Posterior means and medians (in brackets).

\begin{tabular}{l|cccc}
\hline Prior & $\alpha$ & $\lambda$ & $\gamma$ & $R$ \\
\hline Gamma(.001,.001) & $1.203(1.204)$ & $.042(.042)$ & $.012(.012)$ & $.226(.226)$ \\
$\mathrm{U}(0,100)$ & $1.198(1.199)$ & $.043(.043)$ & $.013(.013)$ & $.225(.225)$ \\
\hline
\end{tabular}




\section{Simulation Study}

A simulation study was carried out to assess the sampling properties of the MLE, the posterior mean and the posterior median.

At each of several parameter values and several sample sizes, 1000 simulation batches were independently generated. For each simulation batch, $X_{1}, \ldots, X_{n}$ and $Y_{1}, \ldots, Y_{m}$ were independently drawn from the $\operatorname{TPBT}(\lambda, \alpha)$ and $\operatorname{TPBT}(\gamma, \alpha)$, respectively. Three values were used for each parameter. These were approximately the MLE and the MLE plus or minus 2 standard errors, based on the calculations from the data analysis in Section 5.

For each simulation batch, the MLE and asymptotic 95\% confidence interval (13) for $R$ were evaluated. In addition, for each batch the Metropolis-Hastings algorithm was used to generate a sequence of 10000 samples from the posterior distribution, using independent $\mathrm{U}(0,100)$ priors. The data analysis of Section 4 showed little sensitivity to the choice of prior, and we used the uniform as a non-informative choice. The first 5000 iterates of each sequence were discarded and the remaining 5000 were assumed to be samples from the stationary posterior distribution of $(\alpha, \lambda, \gamma)^{\prime}$. These were transformed to give samples from the posterior distribution of $R$.

Based on the 1000 simulation batches, estimates of the bias and root mean squared error were made for the MLE, the posterior mean and the posterior median. The empirical coverage probabilities were estimated for the asymptotic $95 \%$ confidence interval and the equal tail probability .95 credible interval, the latter being the interval between the $2.5^{\prime}$ th and $97.5^{\prime}$ th percentiles of the empirical posterior distribution. The results are presented in Table 3.

The Bernstein-von Mises' theorem guarantees that for any choice of prior, the MLE, the posterior mean and the posterior median will be close for large sample sizes. In the present case there are only small differences among the MLE, the posterior mean and median with respect to bias and mean squared error, even at small to moderate sample sizes. The coverage property of the frequentist intervals is close to nominal, even when $m=n=20$.

\section{Conclusion}

For the situation when two independent random variables follow the TPBT distribution with equal second shape parameter, the reliability has a particularly simple form, although it still must be estimated numerically. We have examined the use of both likelihood and Bayesian inference for the reliability in this case. Uniform and gamma priors were proposed, and MCMC methods were used to examine the posterior density. Point estimates via maximum likelihood, posterior mean and posterior median all displayed excellent sampling properties. Asymptotic confidence intervals gave coverage close to the nominal level.

There is no guarantee that Bayesian inferences will have frequentist validity. This is the case in Table 3 which shows that the probability .95 Bayesian credible intervals for R have frequentist coverage below $95 \%$. There have been systematic attempts to develop families of prior distributions, so-called probability matching priors, having frequentist validity (Datta, 1996).

A typical adjunct to a Bayesian analysis involving samples generated with MCMC algorithms is the assessment of the stationarity of the sampled points. A number of methods for this purpose are discussed, for example, in Gelman et al (2009). Using several such procedures, there was no indication from the data analysis that more than 10000 points were needed for convergence, using either uniform or gamma priors. Due to the size of the simulation study, we made no assessment of convergence of the sampled points for the individual simulated data sets.

In future work, we will examine the effect of relaxing two assumptions made in this study: the equality of the second shape parameter of the TPBT distribution, and the independence of the two random variables. 
Table 3. Sampling properties of estimators. Pmean - posterior mean. Pmed - posterior median.

$\mathrm{CP}$ - coverge probability. RMS - root mean squared error.

\begin{tabular}{|c|c|c|c|c|c|c|c|c|c|c|c|}
\hline \multicolumn{12}{|c|}{$\mathrm{n}=20, \mathrm{~m}=20$} \\
\hline \multirow[b]{2}{*}{$\alpha$} & \multirow[b]{2}{*}{$\lambda$} & \multirow[b]{2}{*}{$\gamma$} & & \multicolumn{2}{|c|}{ MLE } & \multicolumn{2}{|c|}{ Pmean } & \multicolumn{2}{|c|}{ Pmed } & \multicolumn{2}{|c|}{$\mathrm{CP}$} \\
\hline & & & $R$ & bias & RMS & bias & RMS & bias & RMS & MLE & Bayes \\
\hline 1.100 & 0.020 & 0.0005 & 0.024 & -0.0004 & 0.0139 & 0.0021 & 0.0145 & -0.0002 & 0.0140 & 0.95 & 0.89 \\
\hline 1.100 & 0.020 & 0.0100 & 0.333 & -0.0043 & 0.0761 & -0.0020 & 0.0751 & -0.0041 & 0.0759 & 0.93 & 0.82 \\
\hline 1.100 & 0.020 & 0.0200 & 0.500 & -0.0009 & 0.0824 & -0.0008 & 0.0813 & -0.0009 & 0.0822 & 0.94 & 0.82 \\
\hline 1.100 & 0.040 & 0.0005 & 0.012 & -0.0002 & 0.0083 & 0.0015 & 0.0089 & -0.0001 & 0.0084 & 0.95 & 0.90 \\
\hline 1.100 & 0.040 & 0.0100 & 0.200 & -0.0038 & 0.0609 & -0.0001 & 0.0604 & -0.0032 & 0.0608 & 0.94 & 0.80 \\
\hline 1.100 & 0.040 & 0.0200 & 0.333 & -0.0033 & 0.0796 & -0.0010 & 0.0785 & -0.0030 & 0.0794 & 0.93 & 0.79 \\
\hline 1.100 & 0.060 & 0.0005 & 0.008 & 0.0001 & 0.0061 & 0.0015 & 0.0067 & 0.0002 & 0.0062 & 0.95 & 0.91 \\
\hline 1.100 & 0.060 & 0.0100 & 0.143 & -0.0049 & 0.0490 & -0.0010 & 0.0487 & -0.0043 & 0.0489 & 0.95 & 0.82 \\
\hline 1.100 & 0.060 & 0.0200 & 0.250 & -0.0043 & 0.0657 & -0.0010 & 0.0650 & -0.0038 & 0.0656 & 0.94 & 0.83 \\
\hline 1.200 & 0.020 & 0.0005 & 0.024 & -0.0002 & 0.0137 & 0.0023 & 0.0144 & -0.0000 & 0.0139 & 0.95 & 0.90 \\
\hline 1.200 & 0.020 & 0.0100 & 0.333 & -0.0070 & 0.0763 & -0.0046 & 0.0751 & -0.0067 & 0.0761 & 0.94 & 0.82 \\
\hline 1.200 & 0.020 & 0.0200 & 0.500 & 0.0015 & 0.0876 & 0.0016 & 0.0864 & 0.0014 & 0.0874 & 0.90 & 0.78 \\
\hline 1.200 & 0.040 & 0.0005 & 0.012 & -0.0004 & 0.0080 & 0.0013 & 0.0085 & -0.0003 & 0.0081 & 0.95 & 0.91 \\
\hline 1.200 & 0.040 & 0.0100 & 0.200 & -0.0054 & 0.0593 & -0.0018 & 0.0586 & -0.0050 & 0.0591 & 0.94 & 0.83 \\
\hline 1.200 & 0.040 & 0.0200 & 0.333 & 0.0005 & 0.0789 & 0.0028 & 0.0780 & 0.0009 & 0.0787 & 0.93 & 0.80 \\
\hline 1.200 & 0.060 & 0.0005 & 0.008 & 0.0001 & 0.0065 & 0.0015 & 0.0071 & 0.0002 & 0.0066 & 0.94 & 0.91 \\
\hline 1.200 & 0.060 & 0.0100 & 0.143 & -0.0071 & 0.0498 & -0.0032 & 0.0494 & -0.0065 & 0.0498 & 0.94 & 0.82 \\
\hline 1.200 & 0.060 & 0.0200 & 0.250 & -0.0032 & 0.0673 & 0.0001 & 0.0666 & -0.0026 & 0.0672 & 0.93 & 0.82 \\
\hline 1.300 & 0.020 & 0.0005 & 0.024 & -0.0005 & 0.0144 & 0.0020 & 0.0150 & -0.0003 & 0.0145 & 0.94 & 0.88 \\
\hline 1.300 & 0.020 & 0.0100 & 0.333 & -0.0075 & 0.0771 & -0.0050 & 0.0760 & -0.0071 & 0.0769 & 0.93 & 0.80 \\
\hline 1.300 & 0.020 & 0.0200 & 0.500 & 0.0064 & 0.0813 & 0.0065 & 0.0802 & 0.0065 & 0.0812 & 0.93 & 0.82 \\
\hline 1.300 & 0.040 & 0.0005 & 0.012 & -0.0002 & 0.0082 & 0.0015 & 0.0088 & -0.0001 & 0.0083 & 0.94 & 0.91 \\
\hline 1.300 & 0.040 & 0.0100 & 0.200 & -0.0057 & 0.0611 & -0.0021 & 0.0606 & -0.0052 & 0.0610 & 0.94 & 0.82 \\
\hline 1.300 & 0.040 & 0.0200 & 0.333 & -0.0047 & 0.0775 & -0.0022 & 0.0764 & -0.0042 & 0.0773 & 0.94 & 0.80 \\
\hline 1.300 & 0.060 & 0.0005 & 0.008 & 0.0001 & 0.0062 & 0.0015 & 0.0068 & 0.0002 & 0.0062 & 0.94 & 0.90 \\
\hline 1.300 & 0.060 & 0.0100 & 0.143 & -0.0024 & 0.0482 & 0.0015 & 0.0482 & -0.0018 & 0.0482 & 0.95 & 0.83 \\
\hline 1.300 & 0.060 & 0.0200 & 0.250 & -0.0024 & 0.0655 & 0.0009 & 0.0648 & -0.0019 & 0.0653 & 0.94 & 0.83 \\
\hline \multicolumn{12}{|c|}{$\mathrm{n}=20, \mathrm{~m}=40$} \\
\hline 1.100 & 0.020 & 0.0005 & 0.024 & -0.0003 & 0.0112 & 0.0011 & 0.0116 & -0.0001 & 0.0113 & 0.94 & 0.83 \\
\hline 1.100 & 0.020 & 0.0100 & 0.333 & -0.0041 & 0.0639 & -0.0017 & 0.0634 & -0.0035 & 0.0639 & 0.95 & 0.83 \\
\hline 1.100 & 0.020 & 0.0200 & 0.500 & -0.0042 & 0.0739 & -0.0032 & 0.0732 & -0.0036 & 0.0738 & 0.93 & 0.81 \\
\hline 1.100 & 0.040 & 0.0005 & 0.012 & -0.0001 & 0.0065 & 0.0008 & 0.0068 & 0.0000 & 0.0065 & 0.94 & 0.81 \\
\hline 1.100 & 0.040 & 0.0100 & 0.200 & -0.0074 & 0.0500 & -0.0043 & 0.0496 & -0.0067 & 0.0500 & 0.95 & 0.82 \\
\hline 1.100 & 0.040 & 0.0200 & 0.333 & -0.0066 & 0.0641 & -0.0042 & 0.0634 & -0.0059 & 0.0640 & 0.94 & 0.83 \\
\hline 1.100 & 0.060 & 0.0005 & 0.008 & -0.0001 & 0.0045 & 0.0006 & 0.0048 & -0.0000 & 0.0046 & 0.95 & 0.84 \\
\hline 1.100 & 0.060 & 0.0100 & 0.143 & -0.0042 & 0.0402 & -0.0012 & 0.0402 & -0.0036 & 0.0402 & 0.95 & 0.82 \\
\hline 1.100 & 0.060 & 0.0200 & 0.250 & -0.0034 & 0.0564 & -0.0004 & 0.0560 & -0.0026 & 0.0563 & 0.95 & 0.83 \\
\hline 1.200 & 0.020 & 0.0005 & 0.024 & -0.0012 & 0.0109 & 0.0002 & 0.0112 & -0.0010 & 0.0109 & 0.94 & 0.82 \\
\hline 1.200 & 0.020 & 0.0100 & 0.333 & -0.0104 & 0.0643 & -0.0080 & 0.0634 & -0.0097 & 0.0641 & 0.94 & 0.83 \\
\hline 1.200 & 0.020 & 0.0200 & 0.500 & -0.0035 & 0.0687 & -0.0026 & 0.0680 & -0.0030 & 0.0686 & 0.94 & 0.83 \\
\hline 1.200 & 0.040 & 0.0005 & 0.012 & 0.0000 & 0.0069 & 0.0010 & 0.0073 & 0.0002 & 0.0070 & 0.93 & 0.81 \\
\hline 1.200 & 0.040 & 0.0100 & 0.200 & -0.0085 & 0.0497 & -0.0054 & 0.0492 & -0.0078 & 0.0497 & 0.96 & 0.82 \\
\hline 1.200 & 0.040 & 0.0200 & 0.333 & -0.0091 & 0.0654 & -0.0066 & 0.0647 & -0.0084 & 0.0653 & 0.94 & 0.82 \\
\hline 1.200 & 0.060 & 0.0005 & 0.008 & 0.0001 & 0.0049 & 0.0008 & 0.0052 & 0.0002 & 0.0050 & 0.94 & 0.83 \\
\hline 1.200 & 0.060 & 0.0100 & 0.143 & -0.0045 & 0.0396 & -0.0014 & 0.0396 & -0.0038 & 0.0397 & 0.95 & 0.82 \\
\hline 1.200 & 0.060 & 0.0200 & 0.250 & -0.0046 & 0.0581 & -0.0017 & 0.0576 & -0.0039 & 0.0580 & 0.93 & 0.81 \\
\hline 1.300 & 0.020 & 0.0005 & 0.024 & -0.0005 & 0.0110 & 0.0009 & 0.0114 & -0.0003 & 0.0110 & 0.95 & 0.84 \\
\hline 1.300 & 0.020 & 0.0100 & 0.333 & -0.0078 & 0.0686 & -0.0053 & 0.0680 & -0.0071 & 0.0686 & 0.92 & 0.79 \\
\hline 1.300 & 0.020 & 0.0200 & 0.500 & -0.0023 & 0.0723 & -0.0015 & 0.0715 & -0.0019 & 0.0721 & 0.93 & 0.79 \\
\hline 1.300 & 0.040 & 0.0005 & 0.012 & -0.0003 & 0.0063 & 0.0007 & 0.0066 & -0.0001 & 0.0063 & 0.95 & 0.84 \\
\hline 1.300 & 0.040 & 0.0100 & 0.200 & -0.0042 & 0.0494 & -0.0010 & 0.0492 & -0.0034 & 0.0494 & 0.94 & 0.83 \\
\hline 1.300 & 0.040 & 0.0200 & 0.333 & -0.0029 & 0.0631 & -0.0005 & 0.0625 & -0.0022 & 0.0630 & 0.95 & 0.82 \\
\hline 1.300 & 0.060 & 0.0005 & 0.008 & -0.0001 & 0.0046 & 0.0006 & 0.0049 & -0.0000 & 0.0047 & 0.94 & 0.83 \\
\hline 1.300 & 0.060 & 0.0100 & 0.143 & -0.0039 & 0.0401 & -0.0008 & 0.0401 & -0.0032 & 0.0401 & 0.96 & 0.83 \\
\hline 1.300 & 0.060 & 0.0200 & 0.250 & -0.0061 & 0.0580 & -0.0031 & 0.0575 & -0.0053 & 0.0579 & 0.94 & 0.80 \\
\hline
\end{tabular}


Table 3. Continued ...

\begin{tabular}{|c|c|c|c|c|c|c|c|c|c|c|c|}
\hline \multicolumn{12}{|c|}{$\mathrm{n}=40, \mathrm{~m}=40$} \\
\hline \multirow[b]{2}{*}{$\alpha$} & \multirow[b]{2}{*}{$\lambda$} & \multirow[b]{2}{*}{$\gamma$} & & \multicolumn{2}{|c|}{ MLE } & \multicolumn{2}{|c|}{ Pmean } & \multicolumn{2}{|c|}{ Pmed } & \multicolumn{2}{|c|}{$\mathrm{CP}$} \\
\hline & & & $R$ & bias & RMS & bias & RMS & bias & RMS & MLE & Bayes \\
\hline 1.100 & 0.020 & 0.0005 & 0.024 & -0.0004 & 0.0098 & 0.0007 & 0.0100 & -0.0003 & 0.0098 & 0.94 & 0.83 \\
\hline 1.100 & 0.020 & 0.0100 & 0.333 & -0.0017 & 0.0537 & -0.0004 & 0.0534 & -0.0015 & 0.0537 & 0.94 & 0.82 \\
\hline 1.100 & 0.020 & 0.0200 & 0.500 & -0.0025 & 0.0577 & -0.0024 & 0.0573 & -0.0025 & 0.0576 & 0.95 & 0.82 \\
\hline 1.100 & 0.040 & 0.0005 & 0.012 & -0.0001 & 0.0056 & 0.0007 & 0.0058 & 0.0000 & 0.0056 & 0.96 & 0.84 \\
\hline 1.100 & 0.040 & 0.0100 & 0.200 & -0.0003 & 0.0420 & 0.0016 & 0.0419 & -0.0000 & 0.0419 & 0.95 & 0.82 \\
\hline 1.100 & 0.040 & 0.0200 & 0.333 & -0.0029 & 0.0537 & -0.0017 & 0.0533 & -0.0027 & 0.0537 & 0.94 & 0.82 \\
\hline 1.100 & 0.060 & 0.0005 & 0.008 & -0.0001 & 0.0041 & 0.0005 & 0.0043 & -0.0000 & 0.0041 & 0.94 & 0.85 \\
\hline 1.100 & 0.060 & 0.0100 & 0.143 & -0.0025 & 0.0362 & -0.0006 & 0.0362 & -0.0022 & 0.0362 & 0.92 & 0.80 \\
\hline 1.100 & 0.060 & 0.0200 & 0.250 & -0.0019 & 0.0459 & -0.0003 & 0.0457 & -0.0017 & 0.0458 & 0.95 & 0.83 \\
\hline 1.200 & 0.020 & 0.0005 & 0.024 & -0.0006 & 0.0096 & 0.0005 & 0.0098 & -0.0004 & 0.0096 & 0.94 & 0.84 \\
\hline 1.200 & 0.020 & 0.0100 & 0.333 & -0.0026 & 0.0545 & -0.0013 & 0.0541 & -0.0024 & 0.0544 & 0.94 & 0.81 \\
\hline 1.200 & 0.020 & 0.0200 & 0.500 & -0.0025 & 0.0603 & -0.0023 & 0.0598 & -0.0024 & 0.0602 & 0.93 & 0.80 \\
\hline 1.200 & 0.040 & 0.0005 & 0.012 & -0.0002 & 0.0055 & 0.0005 & 0.0058 & -0.0001 & 0.0056 & 0.95 & 0.84 \\
\hline 1.200 & 0.040 & 0.0100 & 0.200 & -0.0025 & 0.0437 & -0.0006 & 0.0435 & -0.0021 & 0.0437 & 0.93 & 0.82 \\
\hline 1.200 & 0.040 & 0.0200 & 0.333 & -0.0040 & 0.0533 & -0.0027 & 0.0528 & -0.0038 & 0.0532 & 0.94 & 0.82 \\
\hline 1.200 & 0.060 & 0.0005 & 0.008 & -0.0000 & 0.0042 & 0.0005 & 0.0044 & 0.0000 & 0.0043 & 0.94 & 0.83 \\
\hline 1.200 & 0.060 & 0.0100 & 0.143 & -0.0014 & 0.0353 & 0.0006 & 0.0353 & -0.0010 & 0.0352 & 0.94 & 0.81 \\
\hline 1.200 & 0.060 & 0.0200 & 0.250 & -0.0011 & 0.0471 & 0.0006 & 0.0469 & -0.0009 & 0.0471 & 0.94 & 0.83 \\
\hline 1.300 & 0.020 & 0.0005 & 0.024 & -0.0006 & 0.0098 & 0.0004 & 0.0101 & -0.0005 & 0.0099 & 0.94 & 0.82 \\
\hline 1.300 & 0.020 & 0.0100 & 0.333 & -0.0010 & 0.0516 & 0.0003 & 0.0513 & -0.0008 & 0.0516 & 0.95 & 0.83 \\
\hline 1.300 & 0.020 & 0.0200 & 0.500 & -0.0005 & 0.0557 & -0.0003 & 0.0553 & -0.0004 & 0.0556 & 0.96 & 0.82 \\
\hline 1.300 & 0.040 & 0.0005 & 0.012 & -0.0001 & 0.0057 & 0.0007 & 0.0060 & 0.0000 & 0.0058 & 0.96 & 0.84 \\
\hline 1.300 & 0.040 & 0.0100 & 0.200 & -0.0012 & 0.0412 & 0.0007 & 0.0410 & -0.0009 & 0.0412 & 0.95 & 0.82 \\
\hline 1.300 & 0.040 & 0.0200 & 0.333 & -0.0001 & 0.0527 & 0.0012 & 0.0524 & 0.0001 & 0.0527 & 0.95 & 0.81 \\
\hline 1.300 & 0.060 & 0.0005 & 0.008 & 0.0001 & 0.0043 & 0.0007 & 0.0045 & 0.0001 & 0.0043 & 0.94 & 0.82 \\
\hline 1.300 & 0.060 & 0.0100 & 0.143 & -0.0025 & 0.0339 & -0.0005 & 0.0338 & -0.0022 & 0.0339 & 0.96 & 0.84 \\
\hline 1.300 & 0.060 & 0.0200 & 0.250 & -0.0065 & 0.0464 & -0.0048 & 0.0460 & -0.0062 & 0.0463 & 0.95 & 0.82 \\
\hline \multicolumn{12}{|c|}{$\mathrm{n}=40, \mathrm{~m}=60$} \\
\hline 1.100 & 0.020 & 0.0005 & 0.024 & -0.0005 & 0.0083 & 0.0004 & 0.0085 & -0.0004 & 0.0083 & 0.95 & 0.83 \\
\hline 1.100 & 0.020 & 0.0100 & 0.333 & -0.0041 & 0.0469 & -0.0028 & 0.0466 & -0.0037 & 0.0469 & 0.96 & 0.83 \\
\hline 1.100 & 0.020 & 0.0200 & 0.500 & -0.0028 & 0.0524 & -0.0024 & 0.0520 & -0.0027 & 0.0523 & 0.94 & 0.82 \\
\hline 1.100 & 0.040 & 0.0005 & 0.012 & -0.0002 & 0.0048 & 0.0004 & 0.0050 & -0.0001 & 0.0049 & 0.95 & 0.83 \\
\hline 1.100 & 0.040 & 0.0100 & 0.200 & -0.0039 & 0.0397 & -0.0022 & 0.0395 & -0.0035 & 0.0397 & 0.94 & 0.81 \\
\hline 1.100 & 0.040 & 0.0200 & 0.333 & -0.0035 & 0.0462 & -0.0022 & 0.0459 & -0.0031 & 0.0462 & 0.95 & 0.84 \\
\hline 1.100 & 0.060 & 0.0005 & 0.008 & -0.0001 & 0.0035 & 0.0003 & 0.0037 & -0.0001 & 0.0036 & 0.94 & 0.82 \\
\hline 1.100 & 0.060 & 0.0100 & 0.143 & -0.0018 & 0.0307 & -0.0000 & 0.0306 & -0.0014 & 0.0307 & 0.94 & 0.84 \\
\hline 1.100 & 0.060 & 0.0200 & 0.250 & -0.0015 & 0.0429 & 0.0001 & 0.0427 & -0.0012 & 0.0429 & 0.95 & 0.82 \\
\hline 1.200 & 0.020 & 0.0005 & 0.024 & -0.0009 & 0.0084 & -0.0001 & 0.0085 & -0.0008 & 0.0084 & 0.95 & 0.82 \\
\hline 1.200 & 0.020 & 0.0100 & 0.333 & -0.0038 & 0.0479 & -0.0025 & 0.0476 & -0.0035 & 0.0479 & 0.94 & 0.82 \\
\hline 1.200 & 0.020 & 0.0200 & 0.500 & -0.0033 & 0.0528 & -0.0030 & 0.0524 & -0.0032 & 0.0527 & 0.94 & 0.82 \\
\hline 1.200 & 0.040 & 0.0005 & 0.012 & -0.0001 & 0.0051 & 0.0005 & 0.0053 & -0.0000 & 0.0051 & 0.94 & 0.82 \\
\hline 1.200 & 0.040 & 0.0100 & 0.200 & -0.0029 & 0.0372 & -0.0012 & 0.0370 & -0.0025 & 0.0371 & 0.94 & 0.84 \\
\hline 1.200 & 0.040 & 0.0200 & 0.333 & -0.0039 & 0.0489 & -0.0026 & 0.0486 & -0.0036 & 0.0488 & 0.95 & 0.81 \\
\hline 1.200 & 0.060 & 0.0005 & 0.008 & -0.0003 & 0.0036 & 0.0001 & 0.0037 & -0.0003 & 0.0036 & 0.95 & 0.81 \\
\hline 1.200 & 0.060 & 0.0100 & 0.143 & -0.0021 & 0.0302 & -0.0004 & 0.0301 & -0.0018 & 0.0302 & 0.95 & 0.84 \\
\hline 1.200 & 0.060 & 0.0200 & 0.250 & -0.0031 & 0.0430 & -0.0015 & 0.0428 & -0.0028 & 0.0430 & 0.95 & 0.82 \\
\hline 1.300 & 0.020 & 0.0005 & 0.024 & -0.0007 & 0.0085 & 0.0002 & 0.0086 & -0.0005 & 0.0085 & 0.96 & 0.82 \\
\hline 1.300 & 0.020 & 0.0100 & 0.333 & -0.0025 & 0.0489 & -0.0012 & 0.0486 & -0.0022 & 0.0488 & 0.94 & 0.81 \\
\hline 1.300 & 0.020 & 0.0200 & 0.500 & 0.0022 & 0.0511 & 0.0026 & 0.0508 & 0.0024 & 0.0511 & 0.94 & 0.83 \\
\hline 1.300 & 0.040 & 0.0005 & 0.012 & 0.0001 & 0.0049 & 0.0006 & 0.0051 & 0.0001 & 0.0049 & 0.94 & 0.84 \\
\hline 1.300 & 0.040 & 0.0100 & 0.200 & -0.0031 & 0.0387 & -0.0013 & 0.0386 & -0.0027 & 0.0387 & 0.94 & 0.82 \\
\hline 1.300 & 0.040 & 0.0200 & 0.333 & -0.0044 & 0.0474 & -0.0031 & 0.0471 & -0.0041 & 0.0474 & 0.95 & 0.83 \\
\hline 1.300 & 0.060 & 0.0005 & 0.008 & -0.0000 & 0.0038 & 0.0004 & 0.0039 & 0.0000 & 0.0038 & 0.94 & 0.82 \\
\hline 1.300 & 0.060 & 0.0100 & 0.143 & -0.0024 & 0.0304 & -0.0006 & 0.0303 & -0.0020 & 0.0304 & 0.94 & 0.83 \\
\hline 1.300 & 0.060 & 0.0200 & 0.250 & -0.0035 & 0.0424 & -0.0019 & 0.0421 & -0.0031 & 0.0423 & 0.94 & 0.83 \\
\hline
\end{tabular}




\section{References}

Ali, S. (2013). On the Mean Residual Life Function and Stress and Strength Analysis under Different Loss Function for Lindley, Journal of Quality and Reliability Engineering.

http://www.hindawi.com/journals/jqre/2013/190437/ref/

Aykroyd, R. (1998). Bayesian estimation for homogeneous and inhomogeneous Gaussian random fields, IEEE Trans. Pattern Analysis Mach. Intell., 20(5), 533-539. http://dx.doi.org/10.1109/34.682182

Badar, M. G., \& Priest, A. M. (1982). Statistical aspects of fiber and bundle strength in hybrid composites. Progress in Science and Engineering Composites, Hayashi, T., Kawata, K. and Umekawa, S. (eds.), ICCM-IV, Tokyo, 1129 - 1136.

Chen, Z. (2000). A new two-parameter lifetime distribution with bathtub shape or increasing failure rate function. Statistics and Probability Letters, 49, 155-161. http://dx.doi.org/10.1016/S0167-7152(00)00044-4

Church, J. D., \& Harris, B. (1970). The estimation of reliability from stress strength relationships. Technometrics, 12, 49-54. http://dx.doi.org/10.1080/00401706.1970.10488633

Datta, G. S. (1996). On priors providing frequentist validity of Bayesian inference for multiple parametric functions. Biometrika, 83(2), 287-298. http://dx.doi.org/10.1093/biomet/83.2.287

Downton, F. (1973). The estimation of $\operatorname{Pr}(Y<X)$ in the normal case. Technometrics, 15(3), 551-558. http://dx.doi.org/10.2307/1266860

Hastings, W. K. (1970). Monte Carlo sampling methods using Markov chains and their applications. Biometrika, 57(1) 97-109. http://dx.doi.org/10.1093/biomet/57.1.97

Gelman, A., Carlin, J. B., Stern, H. S., \& Rubin, D. R. (2009). Bayesian Data Analysis, Second Edition, Chapman and Hall.

Gilks, W., Richardson, S., \& Spiegelhalter, D. (1996). Markov chain Monte Carlo in Practice, Chapman \& Hall, London.

Gurvich, M. R., Dibenedetto, A. T., \& Rande, S. V. (1997). A new statistical distribution for characterizing the random strength of brittle materials, Journal of Material Science, 32, 2559-2564. http://dx.doi.org/10.1023/A:1018594215963

Kakade, C. S., Shirke, D. T., \& Kundu, D. (2008). Inference for $P(Y<X)$ in Exponentiated Gumbel Distribution. J. Stat. E Appl., 3(1-2), 121-133.

Kumar, K., Krishna, H., \& Garg, R. (2014). Estimation of $P(Y<X)$ in Lindley distribution using progressively first failure censoring. Int J Syst Assur Eng Manag, http://link.springer.com/article/10.1007/s13198-014-0267-9

Kundu, D., \& Gupta, R. D. (2005). Estimation of $P(Y<X)$ for generalized exponential distribution. Metrika, 61(3), 291-308. http://dx.doi.org/10.1007/s001840400345

Kundu, D., \& Gupta, R. D. (2006). Estimation of $P(Y<X)$ for Weibull Distributions. IEEE Transactions on Reliability, 55(2), 270-280. http://dx.doi.org/10.1109/TR.2006.874918

Lecam, L. (1986). Asymptotic Methods in Statistical Decision Theory, Springer-Verlag.

Rezaei, S., Tahmasbi, R., \& Mahmoodi, M. (2010). Estimation of $P(Y<X)$ for generalized Pareto distribution. Journal of Statistical Planning and Inference, 140(2), 480-494. http://www.sciencedirect.com/science/article/pii/S0378375809002432

Sarhan, A. M., Hamilton, D. C., \& Smith B. (2012). Parameter estimation for a two-parameter bathtub-shaped lifetime distribution. Applied Mathematical Modelling, 36(11), 5380-5392.

Sharma, V. K., Singh, S. K., Singh, U., \& Agiwal, V. (2010). The inverse Lindley distribution: A stress-strength reliability model. http://arxiv.org/abs/1405.6268

Tierney, L. (1994). Markov chains for exploring posterior distributions, Ann. Stat., 22(4) 1701-1762. 


\section{Appendix}

The second partial derivatives of $\mathcal{L}(\theta)$ are

$$
\begin{aligned}
\frac{\partial^{2} \mathcal{L}}{\partial \lambda^{2}}= & -\frac{n}{\lambda^{2}}, \quad \frac{\partial^{2} \mathcal{L}}{\partial \gamma^{2}}=-\frac{m}{\gamma^{2}}, \\
\frac{\partial^{2} \mathcal{L}}{\partial \alpha^{2}}= & -\frac{n+m}{\alpha^{2}}+\sum_{i=1}^{n} x_{i}^{\alpha} \ln ^{2} x_{i}+\sum_{i=1}^{m} y_{i}^{\alpha} \ln ^{2} y_{i} \\
& -\lambda \sum_{i=1}^{n}\left(1+x_{i}^{\alpha}\right) e^{x_{i}^{\alpha}} x_{i}^{\alpha} \ln ^{2} x_{i}-\gamma \sum_{i=1}^{m}\left(1+y_{i}^{\alpha}\right) e^{y_{i}^{\alpha}} y_{i}^{\alpha} \ln ^{2} y_{i}, \\
\frac{\partial^{2} \mathcal{L}}{\partial \lambda \partial \alpha}= & -\sum_{i=1}^{n} e^{x_{i}^{\alpha}} x_{i}^{\alpha} \ln x_{i} \\
\frac{\partial^{2} \mathcal{L}}{\partial \gamma \partial \alpha}= & -\sum_{i=1}^{m} e^{y_{i}^{\alpha}} y_{i}^{\alpha} \ln y_{i} \\
\frac{\partial^{2} \mathcal{L}}{\partial \gamma \partial \lambda}= & 0 .
\end{aligned}
$$

The sample information matrix is

$$
\hat{\mathfrak{I}}=-\left(\begin{array}{ccc}
\frac{\partial^{2} \mathcal{L}}{\partial \alpha^{2}} & \frac{\partial^{2} \mathcal{L}}{\partial \alpha \partial \lambda} & \frac{\partial^{2} \mathcal{L}}{\partial \alpha \partial \gamma} \\
\frac{\partial^{2} \mathcal{L}}{\partial \alpha \partial \lambda} & \frac{\partial^{\mathcal{L}} \mathcal{L}}{\partial \lambda^{2}} & \frac{\partial^{2} \mathcal{L}}{\partial \lambda \partial \gamma} \\
\frac{\partial^{2} \mathcal{L}}{\partial \alpha \partial \gamma} & \frac{\partial^{2} \mathcal{L}}{\partial \lambda \partial \gamma} & \frac{\partial^{2} \mathcal{L}}{\partial \gamma^{2}}
\end{array}\right)
$$

The local estimate of the variance-covariance matrix for the MLE of the model parameters is the inverse of the observed information matrix

$$
\hat{\mathfrak{I}}^{-1}=\left(\begin{array}{ccc}
\widehat{\operatorname{var}}(\hat{\alpha}) & \widehat{\operatorname{cov}}(\hat{\alpha}, \hat{\lambda}) & \widehat{\operatorname{cov}}(\hat{\alpha}, \hat{\gamma}) \\
\widehat{\operatorname{cov}}(\hat{\alpha}, \hat{\lambda}) & \widehat{\operatorname{var}}(\hat{\lambda}) & \widehat{\operatorname{cov}}(\hat{\lambda}, \hat{\gamma}) \\
\widehat{\operatorname{cov}}(\hat{\alpha}, \hat{\gamma}) & \widehat{\operatorname{cov}}(\hat{\lambda}, \hat{\gamma}) & \widehat{\operatorname{var}}(\hat{\gamma})
\end{array}\right)=-\left(\begin{array}{ccc}
\frac{\partial^{2} \mathcal{L}}{\partial \alpha^{2}} & \frac{\partial^{2} \mathcal{L}}{\partial \alpha \partial \lambda} & \frac{\partial^{2} \mathcal{L}}{\partial \alpha \partial \gamma} \\
\frac{\partial^{2} \mathcal{L}}{\partial \alpha \partial \lambda} & \frac{\partial^{2} \mathcal{L}}{\partial \lambda^{2}} & \frac{\partial^{2} \mathcal{L}}{\partial \lambda \partial \gamma} \\
\frac{\partial^{2} \mathcal{L}}{\partial \alpha \partial \gamma} & \frac{\partial^{2} \mathcal{L}}{\partial \lambda \partial \gamma} & \frac{\partial^{2} \mathcal{L}}{\partial \gamma^{2}}
\end{array}\right)^{-1}
$$

where the partial derivatives are evaluated at $\alpha=\hat{\alpha}, \lambda=\hat{\lambda}$ and $\gamma=\hat{\gamma}$.

\section{Copyrights}

Copyright for this article is retained by the author(s), with first publication rights granted to the journal.

This is an open-access article distributed under the terms and conditions of the Creative Commons Attribution license (http://creativecommons.org/licenses/by/3.0/). 